\title{
High UBE2T mRNA expression and its prognostic significance in Ovarian cancer: a study based on data mining
}

zenghong wu ( D D201981596@hust.edu.cn )

\section{Research article}

Keywords: Ovarian cancer, Ubiquitin conjugating enzyme E2 T, data mining, prognosis

Posted Date: January 10th, 2020

DOl: https://doi.org/10.21203/rs.2.20556/v1

License: (c) (i) This work is licensed under a Creative Commons Attribution 4.0 International License.

Read Full License 


\section{Abstract}

Objection

Ovarian cancer (OC) affects approximately 22000 women annually in the US and ranks 5th in cancer deaths, largely due to diagnosed with advanced stage1. Epithelial ovarian cancer (EOC) accounts for approximately $90 \%$ of all ovarian cancer cases. Our study was to assess the prognostic meaningful of UBE2T expression in OC dependent on data acquired from TCGA and so as to increase further knowledge into the biological pathways involved in OC pathogenesis related to UBE2T.

\section{Method}

Information on gene expression and comparing clinical data were recognized and downloaded from TCGA. Gene set enrichment analysis (GSEA) created an arranged list of all genes $s$ indicated by their connection with UBE2T expression.

\section{Results}

The scatter plot showed the difference of UBE2T expression between normal and tumor samples $(P<0.01)$. In order to decide the biological interaction network of UBE2T in OC, we applied to tab Network in cBioPortal and the 50 most as often altered neighbor genes of UBE2T were showed utilizing Network and the most frequent alterations were HES1. The GSEA results showed that cell cycle, DNA replication, RNA degradation, some cancers, spliceosome, Huntington's disease, oxidative phosphorylation are differentially enriched in UBE2T high expression phenotype. Cumulative survive showed that dendritic cell of immune infiltrates statistically significant $(P<0.05)$ of UBE2T in OC indicating that dendritic cell significantly affecting the prognosis, it is worth further research and exploration.

\section{Conclusion}

Our study found that the expression of UBE2T was significantly increased in OC patients and associated with several clinical features. UBE2T may be a potentially useful prognostic molecular biomarker of bad survival in $\mathrm{OC}$, while further experimental ought to be performed to demonstrate the biologic effect of UBE2T.

\section{Background}

Ovarian cancer (OC) affects near 22000 women annually in the US and ranks 5th in cancer deaths, largely due to diagnosed with advanced stage ${ }^{1}$. Epithelial ovarian cancer (EOC) constitute approximately $90 \%$ of all ovarian cancer cases ${ }^{2}$. Despite recent advances in cyto-reductive surgery and chemotherapy, the 5-year survival rate of EOC patients is only $30 \%$ and the prognosis of EOC remains poor. The survival rate in general increases up to $70 \%$ if effective early stage detection is possible. The leading cause of death was by extensive peritoneal metastasis, and the specific molecular mechanism remains unclear ${ }^{3}$. Several aspects influence the progression of the disease. Genetic and epigenetic factors are the most 
important ones among them. Mutations and the loss of the TP53 function are found in $60 \%-80 \%$ of the familial and sporadic cases of the disease ${ }^{4}$. Study reported that ET-1R/ $\beta$-arr1 axis enables cancer cells to engage several integrated signaling in $\mathrm{OC}$ and may be a novel therapeutic approach ${ }^{5}$. Pan et al reported that LINC00339 regulated ovarian cancer cell migration, proliferation and invasion by targeting miR-148a3p/ROCK1 axes. Moreover, a recent study found Rab23 is highly expressed in ovarian cancer tissues and associated with advanced stage, and shortened overall survival time of ovarian cancer patients ${ }^{7}$. Similarly, Wu et al found high expression of SCNN1A was related to poor overall survival and progressionfree survival in ovarian cancer patients ${ }^{8}$. Due to lacking specific and sensitive early biomarkers, a high possibility of metastasis and drug resistance is considered to make contributions the high mortality of $\mathrm{OC}$. In this way, it is necessary for recognizing the more sensitive and specific novel target molecules for developing effective diagnosis and treatment strategies of OC.

Posttranscriptional modifications are vital in the initiation and the progression of tumors. Ubiquitin conjugating enzyme E2 T (UBE2T; otherwise called FANCT; PIG50; HSPC150), is a member of the ubiquitin-proteasome family and encoded a protein catalyzes the covalent attachment of ubiquitin to protein substrates. It was initially reported in Fanconi anemia and is important to DNA damage repair? UBE2T participates in main cellular processes such as signal transduction, cell cycle control and tumorigenesis via triggering the degradation of relevant substrates ${ }^{10}$. Studies reported that UBE2T is amplified in a wide variety of tumors. Ueki et al ${ }^{11}$ implied a critical role of UBE2T in the progression of breast cancer through the regulation and the interaction with the BRCA1/BARD1 complex. Hu et al ${ }^{12}$ demonstrated that UBE2T might promote the development and progression of nasopharyngeal carcinoma by activating the AKT/GSK3 $\beta / \beta$-catenin pathway. Gong et $\mathrm{al}^{13}$ reported that the knockdown of UBE2T significantly diminished bladder cancer cell proliferation and colony formation. Liu et $\mathrm{al}^{14}$ found that miR-543/UBE2T/p53 axis might represent a new significant potential therapeutic target for hepatocellular carcinoma intervention. Luo et al ${ }^{15}$ reported that UBE2T facilitate the tumor invasion and metastasis in gastric cancer via triggered the epithelial mesenchymal transition process. In our study, we have assessed the expression of UBE2T in OC dependent on data acquired from online databases and so as to increase further knowledge into the biological pathways involved in OC pathogenesis related UBE2T.

\section{Methods}

TCGA and GSEA analysis

Information on gene expression were recognized and downloaded from Genotype Tissue Expression (GTEx) projects and The Cancer Genome Atlas (TCGA) via UCSC database (https://genome.ucsc.edu) ${ }^{15}$. Boxplots were utilized to envision expression differences for discrete variables. Gene set enrichment analysis (GSEA) created an arranged list of all genes s indicated by their connection with UBE2T expression. Then samples were divided into high- and low-UBE2T groups as training set to distinguish the potential function and elucidate the significant survival difference utilizing GSEA. Annotated gene sets c2.cp.kegg.v6.0.symbols.gmt was selected as the reference gene sets, which includes terms with FDR< 
0.05. Gene set permutations were executed multiple times for every examination. The expression degree of UBE2T was applied as a phenotype label. The normalized enrichment score (NES) and nominal P value had been used to kind the pathways enriched in every phenotype.

\section{UALCAN Dataset and Kaplan-Meier plotter}

UALCAN ${ }^{16}$ is an intelligence and user-friendly web asset for discovering, analyzing and integrating cancer transcriptome data and in-depth analyses of TCGA gene expression data. The prognostic meaning of mRNA expression of UBE2T in OC was assessed by using Kaplan-Meier plotter ${ }^{17}$, in which data about gene expression with survival of patients in 21 cancer types. In Kaplan-Meier plotter, cancer patient samples were split into low and high expression group according to median values of mRNA expression and assessed by K-M survival plot.

CBioPortal analysis and immune infiltrates analysis

cBioPortal $^{18}$ is an open access asset that visualizes, analyzes and downloads large-scale cancer genomics datasets. The portal currently contains 245 cancer studies. We used c-BioPortal to analyze UBE2T changes in TCGA OV samples and showed an overview of the genetic changes in each test in UBE2T. We constructed a UBI2T tag biointeraction network. TIMER ${ }^{19}$ is a comprehensive asset for systematically studying the immune infiltration of various malignancies. The abundance of six immune infiltrates (CD8 + T cells, B cells, CD $4+T$ cells, macrophages, neutrophils and dendritic cells) was assessed by our statistical methods and pathological estimation methods have been used. It was evaluated.

TargetScan analysis

TargetScan ${ }^{20}$ is a flexible web interface for predicting miRNA biological targets. TargetScanHuman believes that matching to human 3'UTR and its orthologs is characterized by a UCSC genome-wide alignment. FunRich ${ }^{21}$ is an independent programming tool designed to process a variety of gene/protein datasets that are not related to organisms and are used for functional enrichment and interaction of genes and proteins. Network analysis. Currently, TargetScan is used to study differentially expressed miRNAs associated with UBE2T, and then we use the Funrich tool for miRNA enrichment analysis, including biological processes, cellular components, molecular functions, and biological pathways.

\section{Results}

Association with UBE2T expression and clinicopathologic factors

The scatter plot showed the difference of UBE2T expression between normal and tumor samples $(\mathrm{P}<$ $0.01)$, Fig. 1A. We next used UALCAN to explore the relationship of expression of UBE2T and clinical factors. In age subgroup (normal-vs-age (21-40yrs), normal-vs-age (41-60yrs), normal-vs-age (61-80yrs) and normal-vs-age (81-100yrs)) analysis the transcription level of UBE2T was essentially higher in OC 
patients than healthy individuals; race subgroup (normal-vs-Caucasian, normal-vs-AfricanAmerican and normal-vs-Asian); tumor grade subgroup (normal-vs-Grade 1, normal-vs-Grade 2, normal-vs-Grade 3 and normal-vs-Grade 4) analysis the UBE2T was also significantly higher in OC patients Fig. 1B-1E.

GSEA recognizes UBE2T related signaling pathway

In order to recognize signaling pathways which might be differentially initiated in OC, we led GSEA analysis among low and high UBE2T expression data sets (FDR $\mathrm{P}<0.05$, NOM $\mathrm{P}<0.05)$. We chose the most significantly enriched signaling pathways dependent on normalized enrichment score (NES) Table 1. The results show that cell cycle, DNA replication, RNA degradation, some cancers, spliceosome, Huntington's disease, oxidative phosphorylation are differentially enriched in UBE2T high expression phenotype Fig. 2.

Table 1

Gene sets enriched in phenotype high

\begin{tabular}{|lllll|}
\hline Gene Symbol & Amplification & $\begin{array}{l}\text { Homozygous } \\
\text { Deletion }\end{array}$ & Mutation & $\begin{array}{l}\text { Total } \\
\text { Alteration }\end{array}$ \\
\hline HES1 & 13.6 & 0.3 & 0.3 & 14.2 \\
\hline RFC4 & 13.3 & 0 & 0 & 13.3 \\
\hline FAAP24 & 7.9 & 0 & 0 & 7.9 \\
\hline UBE3C & 6.3 & 0.3 & 0 & 6.6 \\
\hline UBE2Q1 & 4.7 & 0 & 0 & 4.7 \\
\hline
\end{tabular}

Survival and cBioPortal results

Overall survival (OS), progression-free survival (PFS) and post-progression survival (PPS) analysis demonstrated that OC with UBE2T -high had a more terrible prognosis than that with UBE2T -low $(\mathrm{P}<$ 0.05) Fig. 3. In order to decide the biological interaction network of UBE2T in OC, we applied to tab Network in cBioPortal and the 50 most as often altered neighbor genes of UBE2T were showed utilizing Network and the most frequent alterations were HES1 (14.2\%) (Fig. 4 and Table 2). 


\begin{tabular}{|c|c|c|c|c|c|}
\hline Gene Symbol & Amplification & $\begin{array}{l}\text { Homozygous } \\
\text { Deletion }\end{array}$ & Mutation & $\begin{array}{l}\text { Total } \\
\text { Alteration }\end{array}$ & \\
\hline HES1 & 13.6 & 0.3 & 0.3 & 14.2 & \\
\hline RFC4 & 13.3 & 0 & 0 & 13.3 & Table 2. The type and \\
\hline FAAP24 & 7.9 & 0 & 0 & 7.9 & frequency of UBE2T \\
\hline UBE3C & 6.3 & 0.3 & 0 & 6.6 & OC (cBioPortal). \\
\hline UBE2Q1 & 4.7 & 0 & 0 & 4.7 & \\
\hline
\end{tabular}

UBE2T in OC

OC expression of UBE2T immune infiltrates (B cells, CD4 + T cells, CD8 + T cells, neutrophils, macrophages, and dendritic cells) correlation between the abundance of statistical significance $(P<0.05$, Fig. 5A). Cumulative survival showed that UBC2T immunosuppressive dendritic cells in OC were statistically significant $(P<0.05)$, indicating that dendritic cells significantly affected prognosis and warrant further study and exploration (Fig. 5B). A box plot was introduced to demonstrate the distribution of each immunization subgroup in each copy number state with UBE2T in OC. According to the cumulative weighted context + + score, the top three of the 1169 miRNA families associated with the gene UBE2T in OC are hsa-miR-5580-3p, hsa-miR-3652 and hsa-miR-4430. Figure 6A shows conserved sites of the widely conserved miRNA family in vertebrates. To examine the function of the identified 1169 miRNAs, bioconcentration was performed by the Funrich database. Biological processes are significantly rich in nucleobase regulation, signal transduction, cellular communication, transport, cell growth, and regulation of cellular tissue and biogenesis. Cellular components are mainly enriched in the nucleus, cytoplasm, Golgi apparatus, endosomes, lysosomes and early endosomes. Molecular functions are mainly enhanced by transcription factor activity, transcriptional regulatory activity, protein serine, GTPase activity and ubiquitin-specific protease activity; biological pathways are abundant, including Glypican pathway, proteoglycan syndecan-mediated signaling events, VEGF and VEGFR signals Network, TRAIL signaling pathway, sphingosine 1-phosphate (S1P) pathway and ErbB signaling pathway Fig. 6B-6E.

\section{Discussion}

In this study, we conducted a comprehensive and detailed assessment of UBE2T expression in ovarian cancer based on online database and to explore its association with clinicopathologic characteristics, survival, function, immune infiltrates and expression difference. Knowing whether a more highlyexpression biomarkers in tumor consists of a right away link to ovarian cancer help us understand the mechanistic clarification for observed clinical survival patterns. In our outcomes, UBE2T significantly expression between normal and tumor samples indicated that UBE2T may plays an important role in regulate cancer progression. 
UBE2T as for a member of E2 the family in the ubiquitin-proteasome pathway, a complex protein degradation system that participated in extensive biological processes, including signal transduction, tumorigenesis, cell proliferation, differentiation and cell cycle control. Numerous studies identified that its overexpression results in a variety of tumorigenesis such as osteosarcoma, diffuse large B-cell lymphoma and malignant pleural mesothelioma $22-24$. Until now, the expression of UBE2T and its potential prognostic effect on $\mathrm{OC}$ has not yet been investigated. Our outcomes showed that $\mathrm{OC}$ with UBE2T -high had a more terrible prognosis than that with UBE2T -low $(P<0.05)$ in OS, PFS and PPS. Moreover, we used UALCAN database to further analysis of multiple clinic pathological features of OC samples and all indicated high transcription of UBE2T. In order to recognize signaling pathways that are differentially in OC, we then performed GSEA analysis among low and high UBE2T expression data sets. The results show that cell cycle, DNA replication, RNA degradation, some cancers, spliceosome, Huntington's disease, oxidative phosphorylation are differentially enriched in UBE2T high expression phenotype. UBE2T may affect cell cycle, DNA replication, RNA degradation then regulates the occurrence and development of cancer cells. Hao et $\mathrm{al}^{25}$ was first to investigate the expression of UBE2T mRNA in normal human tissues and 8 lung cancer cell lines and found UBE2T was significantly upregulated in lung cancer tissue and cell lines, suggesting involvement of UBE2T in the malignant cell phenotype. The ubiquitin-proteasome system exerts a crucial role in extensive biological processes, and UBE2T, its crucial member, may affect tumorigenesis and cell cycle. Studies on the function of UBE2T in cancer will undoubtedly provide new insights regarding the role UBE2T in both cell cycle regulation and tumorigenesis.

In order to decide the biological interaction network of UBE2T in OC, we applied to tab Network in cBioPortal and the 50 most as often altered neighbor genes of CENPM and the most frequent alterations were HES1. Hes family bHLH transcription factor 1 (HES1) belongs to the basic helix-loop-helix family of transcription factors. A recent study found that Hes1 expression oscillates and drives cyclic expression of the proneural gene Ascl1, which activates cell proliferation in active neural stem cells ${ }^{26}$. Study also found that indicating that the Notch1/Hes1/MMPs pathway ST6Gal-can mediate the invasiveness and tumorigenicity of non-small cell lung cancer (NSCLC) cells in vitro and in vivo ${ }^{27}$. Huang et al ${ }^{28}$ demonstrated that HES1 is a specific downstream gene of NOTCH1 and that it contributes to salivary adenoid cystic carcinoma proliferation, apoptosis and metastasis. In addition, Islam et al reported ${ }^{29}$ that cisplatin/eugenol sequential combination could be of great therapeutic value for ovarian cancer patients through targeting the Notch-Hes1 pathway and the consequent elimination of the resistant cancer stem cells. So, our study may provide information for HES1 on replication forks study in OC patients.

Until now, there was no related study try to explore the connection of immune infiltrates and UBE2T, we first study the immune infiltrates and miRNAs in correlation with UBE2T in OC. Correlation between UBE2T in OC expression and abundance of immune infiltrates was statistically significant and the cumulative survive showed that dendritic cell of immune infiltrates statistically significant $(P<0.05)$ of UBE2T in OC indicating that dendritic cell significantly affecting the prognosis, it is worth further research and exploration. We finally study the miRNAs that correlation with UBE2T in OC. The top 3 among 1169 
miRNAs family was hsa-miR-5580-3p, hsa-miR-3652 and hsa-miR-4430 that related to gene UBE2T in OC. Study reported miR-3652 association with oral squamous cell carcinoma prognosis and miR-4430 related to breast cancer cells prognosis ${ }^{30,31}$. To examine the function of the identified 1169 miRNAs, biological enrichment was performed via Funrich database. Biological pathway enriched in glypican pathway, proteoglycan syndecan-mediated signaling events, VEGF and VEGFR signaling network, TRAIL signaling pathway, sphingosine 1-phosphate (S1P) pathway and ErbB signaling pathway. Thus, UBE2T may help us understand the protein disease pathogenesis and progression in future study. Our study found that the expression of UBE2T was significantly increased in OC patients and associated with several clinical features. UBE2T may be a potentially useful prognostic molecular biomarker of bad survival in OC, while further experimental ought to be performed to demonstrate the biologic effect of UBE2T.

\section{Abbreviations}

OC:Ovarian cancer; GSEA:Gene set enrichment analysis; UBE2T:Ubiquitin conjugating enzyme E2 T; GTEx:Genotype Tissue Expression; TCGA:The Cancer Genome Atlas.

\section{Declarations}

\section{Acknowledgements}

Not applicable

\section{Authors' contributions}

W.Z.H. designed and analyzed the research study; W.Z.H. wrote and revised the manuscript, W.Z.H. collected the data and all authors contributed to and approved the final version of manuscript.

\section{Funding}

This work is not supported by grants.

\section{Availability of data and materials}

RNA-seq data and corresponding clinical data were acquired from the data portal for TCGA (https://portal. gdc.cancer.gov/)

\section{Ethics approval and consent to participate}

Not applicable.

\section{Consent for publication}

Not applicable. 


\section{Competing interests}

The authors declare that they have no competing interests.

\section{References}

1. S. Cancer Statistics Working Group. U.S. Cancer Statistics Data Visualizations Tool, based on November 2017 submission data (1999-2015): U.S. Department of Health and Human Services, Centers for Disease Control and Prevention and National Cancer Institute. www.cdc.gov/cancer/dataviz. Accessed January 10, 2019

2. Vargas-Hernandez VM, Moreno-Eutimio MA, Acosta-Altamirano G, Vargas-Aguilar VM. Management of recurrent epithelial ovarian cancer. Gland Surg. 2014;3(3):198-202.

3. Naora H, Montell DJ. Ovarian cancer metastasis: integrating insights from disparate model organisms. Nat Rev Cancer. 2005;5(5):355-366.

4. Wang X, Wang E, Kavanagh JJ, Freedman RS. Ovarian cancer, the coagulation pathway, and inflammation. J Transl Med. 2005;3:25.

5. Tocci P, Rosanò L, Bagnato A. Targeting Endothelin-1 Receptor/ $\beta$-Arrestin-1 Axis in Ovarian Cancer: From Basic Research to a Therapeutic Approach. Front Endocrinol (Lausanne). 2019 Sep 4;10:609.

6. Pan L, Meng Q, Li H et al. LINC00339 promotes cell proliferation, migration, and invasion of ovarian cancer cells via miR-148a-3p/ROCK1 axes. Biomed Pharmacother. 2019 Sep 21;120:109423.

7. Gao L, Zheng M, Guo Q et al. Downregulation of Rab23 inhibits proliferation, invasion, and metastasis of human ovarian cancer. Int J Biochem Cell Biol. 2019 Sep 21;116:105617.

8. Wu L, Ling ZH, Wang H et al. Upregulation of SCNN1A Promotes Cell Proliferation, Migration, and Predicts Poor Prognosis in Ovarian Cancer Through Regulating Epithelial-Mesenchymal Transformation. Cancer Biother Radiopharm. 2019 Sep 24.

9. Alpi A, Langevin F, Mosedale G, Machida YJ, Dutta A and Patel KJ: UBE2T, the fanconi anemia core complex, and Fancd2 are recruited independently to chromatin: a basis for the regulation of Fancd2 monoubiquitination. Mol cell Biol 27: 8421-8430, 2007.

10. H. Lim, M.H. Song, K.H. Baek, Decision for cell fate: deubiquitinating enzymes in cell cycle checkpoint, Cell Mol. Life Sci. 73 (2016) 1439e1455.

11. Ueki, J.H. Park, T. Nishidate, K. Kijima, K. Hirata, Y. Nakamura, T. Katagiri, Ubiquitination and downregulation of BRCA1 by ubiquitin-conjugating enzyme E2T overexpression in human breast cancer cells, Cancer Res. 69 (2009) 8752e8760.

12. Hu, L. Xiao, C. Cao, S. Hua, D. Wu, UBE2T promotes nasopharyngeal carcinoma cell proliferation, invasion, and metastasis by activating the AKT/ GSK3beta/beta-catenin pathway, Oncotarget 7 (2016) $15161 \mathrm{e} 15172$.

13. Gong Y, Peng D, Ning X, et al. UBE2T silencing suppresses proliferation and induces cell cycle arrest and apoptosis in bladder cancer cells[J]. Oncology Letters, 2016. 
14. Liu L P, Yang M, Peng Q Z, et al. UBE2T promotes hepatocellular carcinoma cell growth via ubiquitination of p53. Biochemical and Biophysical Research Communications, 2017:S0006291X17318624.

15. Casper J, Zweig A S, Villarreal C, et al. The UCSC Genome Browser database: 2018 update[J]. Nucleic Acids Research, 2017, 46(Database issue):D762-D769.

16. Tang, Z.; Li, C.; Kang, B.; Gao, G.; Li, C.; Zhang, Z. Gepia: A web server for cancer and normal gene expression profiling and interactive analyses. Nucleic Acids Res. 2017, 45, W98-W102. https://doi.org/10.1093/nar/gkx247

17. Nagy A, Lánczky A, Menyhárt 0 , Győrffy B. Validation of miRNA prognostic power in hepatocellular carcinoma using expression data of independent datasets, Scientific Reports, 2018;8:9227. https://doi.org/10.1038/s41598-018-27521-y

18. Gao et al. Sci. Signal. 2013 \& Cerami et al. Cancer Discov. 2012 when publishing results based on cBioPortal. https://doi.org/10.1158/2159-829

19. Li T, Fan J, Wang B, et al. TIMER: A Web Server for Comprehensive Analysis of Tumor-Infiltrating Immune Cells. Cancer Research, 2017, 77(21):e108-e110.https://doi.org/10.1158/0008-5472.CAN17-0307

20. Agarwal V, Bell GW, Nam J, Bartel DP. Predicting effective microRNA target sites in mammalian mRNAs. eLife, 2015, 4:e05005. https://doi.org/10.7554/eLife.05005.001

21. FunRich: An open access standalone functional enrichment and interaction network analysis tool. PROTEOMICS, 2015, 15(15):2597-2601. https://doi.org/10.1002/pmic.201400515

22. Gordon GJ, Appasani K, Parcells JP, Mukhopadhyay NK, Jaklitsch MT, Richards WG, Sugarbaker DJ, Bueno R: Inhibitor of apoptosis protein-1 promotes tumor cell survival in mesothelioma. Carcinogenesis 2002; 23: 1017-1024.

23. Kosari F, Parker AS, Kube DM, Lohse CM, Leibovich BC, Blute ML, Cheville JC, Vasmatzis G: Clear cell renal cell carcinoma: gene expression analyses identify a potential signature for tumor aggressiveness. Clin Cancer Res 2005; 11: 5128-5139.

24. Wang $Y$, Leng $H$, Chen $H$, Wang L, Jiang $N$, Hua $X$ and Yu B: Knockdown of uBe2T inhibits osteosarcoma cell proliferation, migration, and invasion by suppressing the Pi3K/akt signaling pathway. oncol res 24: 361-369, 2016.

25. Hao J, Xu A, Xie X, et al. Elevated Expression of UBE2T in Lung Cancer Tumors and Cell Lines[J]. Tumor Biology, 2008, 29(3):195-203.

26. Sueda R, Imayoshi I, Harima $Y$ et al. High Hes1 expression and resultant Ascl1 suppression regulate quiescent vs. active neural stem cells in the adult mouse brain. Genes Dev. 2019 May 1;33(9-10):511523.

27. Yuan Q, Chen X, Han Y et al. Modification of a2,6-sialylation mediates the invasiveness and tumorigenicity of non-small cell lung cancer cells in vitro and in vivo via Notch1/Hes1/MMPs pathway. Int J Cancer. 2018 Nov 1;143(9):2319-2330. 
28. Huang $X Y$, Gan R H, Xie J, et al. The oncogenic effects of HES1 on salivary adenoid cystic carcinoma cell growth and metastasis[J]. BMC Cancer, 2018, 18(1):436.

29. Islam SS, Aboussekhra A. Sequential combination of cisplatin with eugenol targets ovarian cancer stem cells through the Notch-Hes1 signalling pathway. J Exp Clin Cancer Res. 2019 Aug 30;38(1):382.

30. Li S, Liu X, Zhou Y et al. Shared genetic and epigenetic mechanisms between chronic periodontitis and oral squamous cell carcinoma. Oral Oncol. 2018 Nov;86:216-224.

31. Tanman Ü, Yangın S, Cansaran-Duman D. Determination of Dysregulated miRNA Expression Levels by qRT-PCR after the Application of Usnic Acid to Breast Cancer. Anticancer Agents Med Chem. 2019 Sep 23.

\section{Figures}
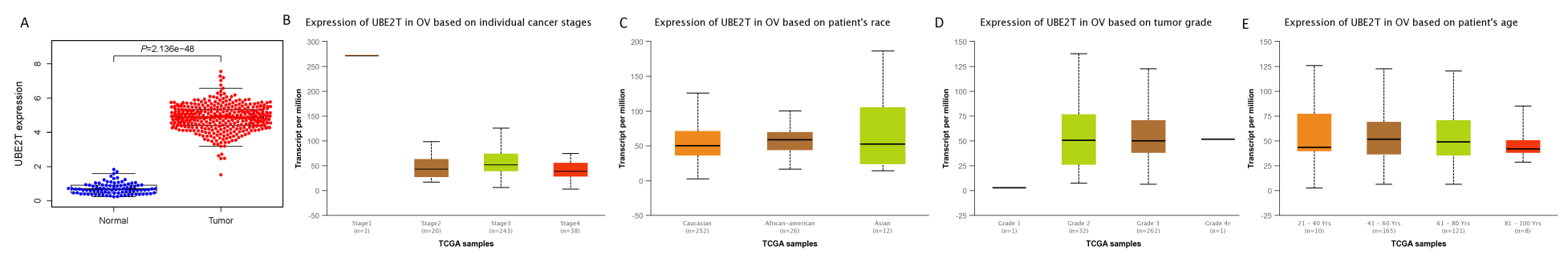

\section{Figure 1}

The scatter plot showed the difference of UBE2T expression between normal and tumor samples $(P<0.01)$, Fig. 1A. We next used UALCAN to explore the relationship of expression of UBE2T and clinical factors. In age subgroup (normal-vs-age (21-40yrs), normal-vs-age (41-60yrs), normal-vs-age (61-80yrs) and normal-vs-age (81-100yrs)) analysis the transcription level of UBE2T was essentially higher in OC patients than healthy individuals; race subgroup (normal-vs-Caucasian, normal-vs-AfricanAmerican and normal-vs-Asian); tumor grade subgroup (normal-vs-Grade 1, normal-vs-Grade 2, normal-vs-Grade 3 and normal-vs-Grade 4) analysis the UBE2T was also significantly higher in OC patients Fig. 1B-1E. 


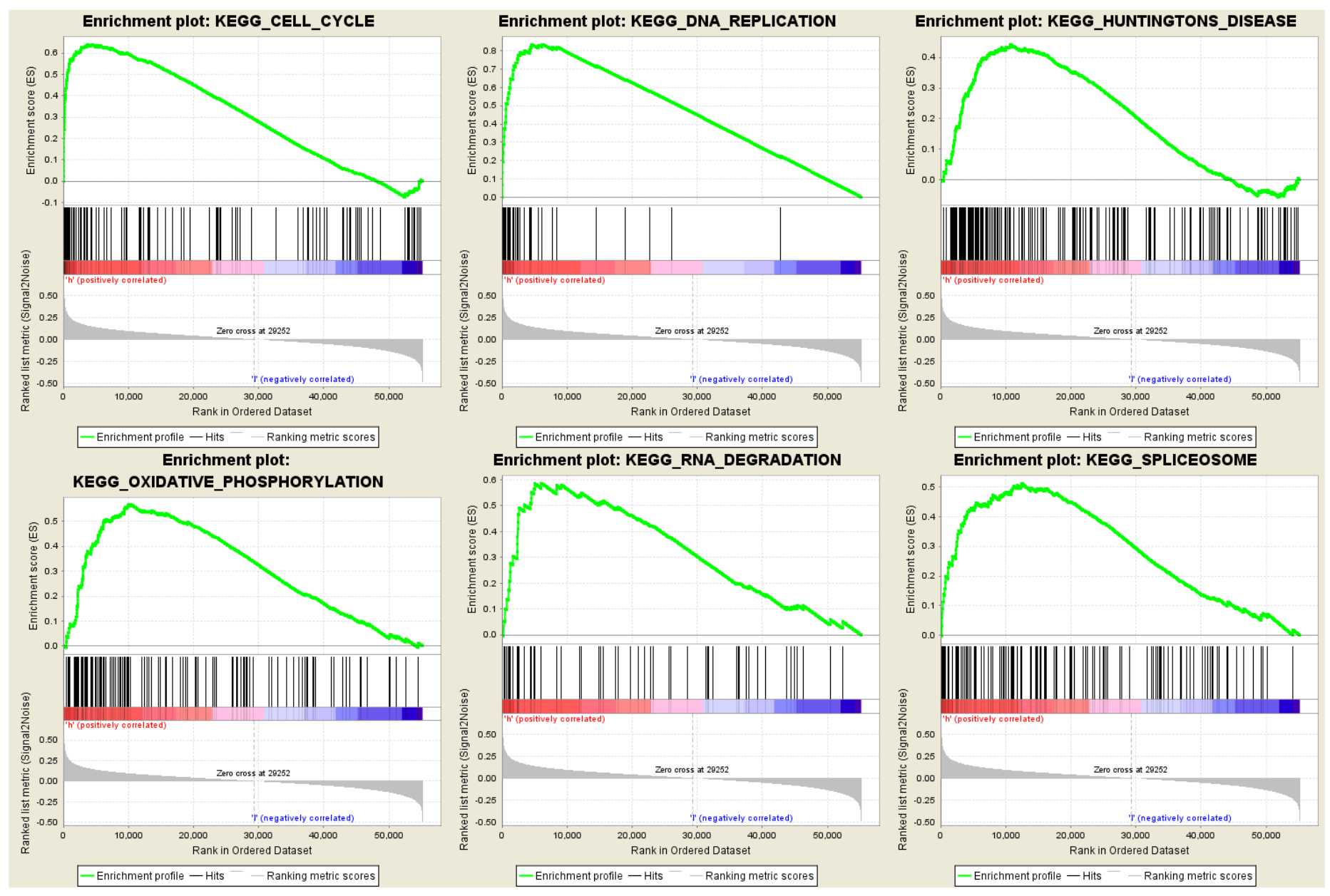

Figure 2

The results show that cell cycle, DNA replication, RNA degradation, some cancers, spliceosome, Huntington's disease, oxidative phosphorylation are differentially enriched in UBE2T high expression phenotype
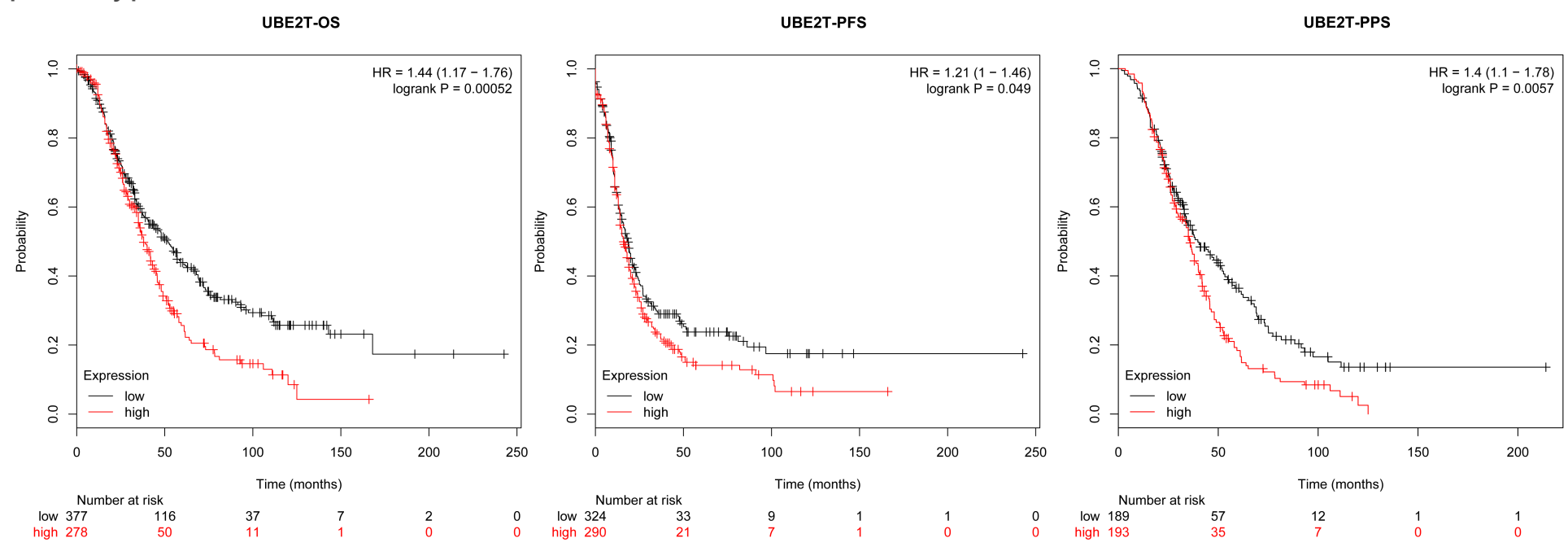

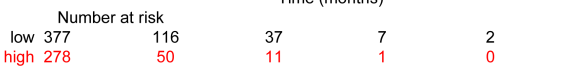

Figure 3 
Overall survival (OS), progression-free survival (PFS) and post-progression survival (PPS) analysis demonstrated that OC with UBE2T -high had a more terrible prognosis than that with UBE2T -low $(P<0.05)$

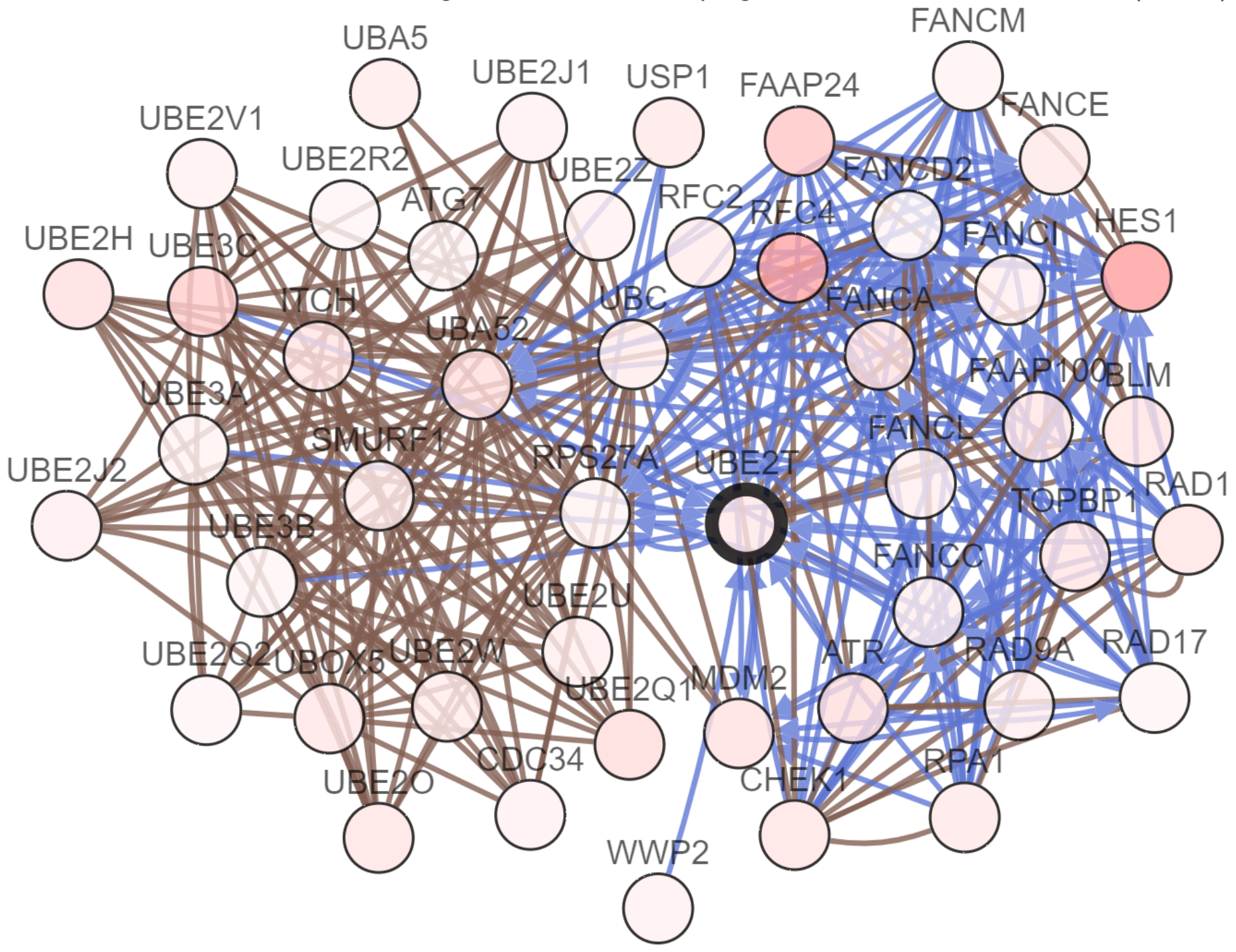

\section{Figure 4}

In order to decide the biological interaction network of UBE2T in OC, we applied to tab Network in cBioPortal and the 50 most as often altered neighbor genes of UBE2T were showed utilizing Network and the most frequent alterations were HES1 (14.2\%) 
A
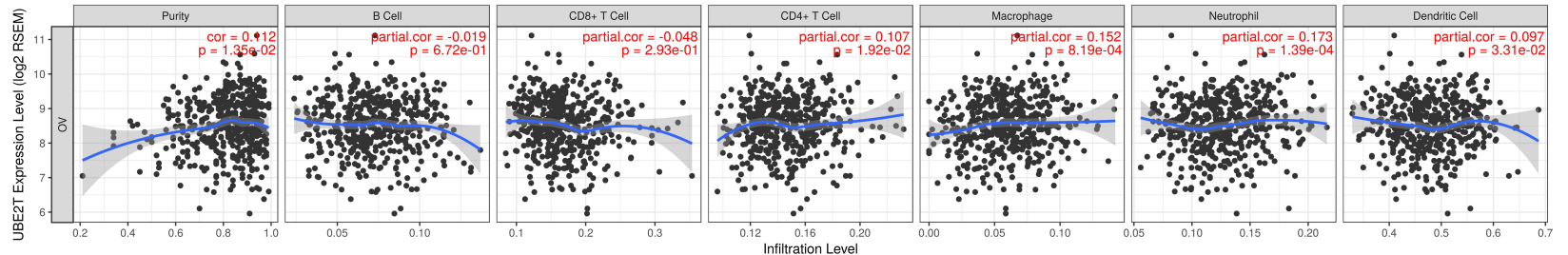

B

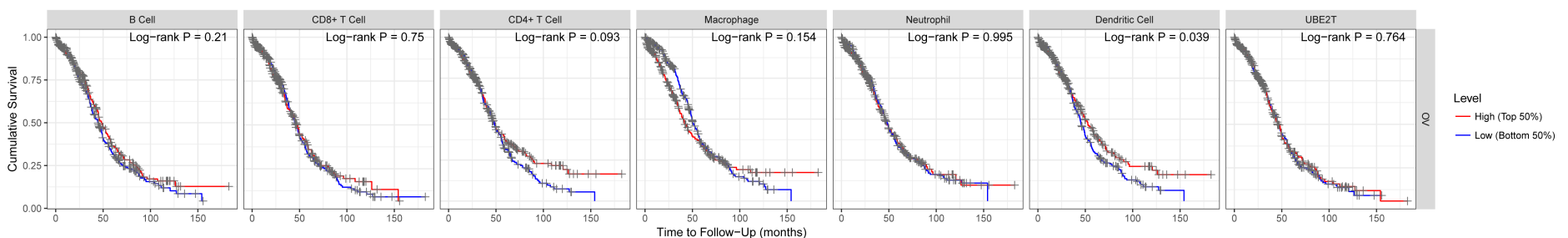

C

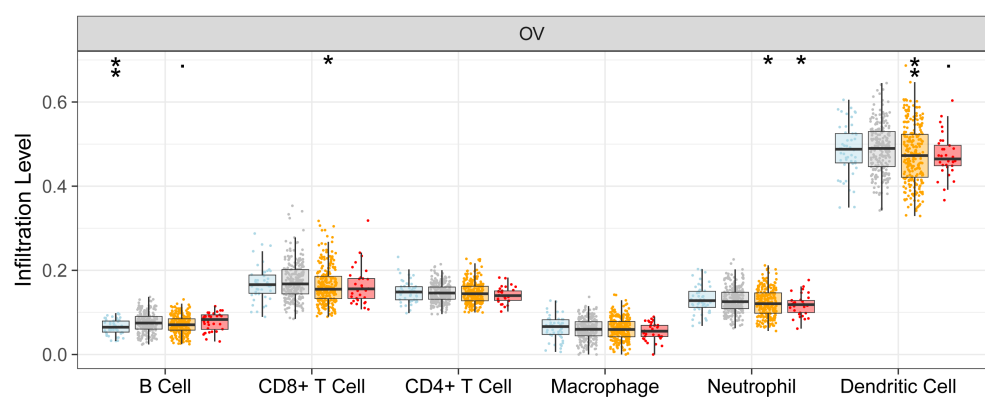

Copy Number

Arm-level Deletion

官 Diploid/Normal

帛 High Amplication

\section{Figure 5}

OC expression of UBE2T immune infiltrates (B cells, CD4 + T cells, CD8 + T cells, neutrophils, macrophages, and dendritic cells) correlation between the abundance of statistical significance $(P<0.05$, Fig. 5A). Cumulative survival showed that UBC2T immunosuppressive dendritic cells in OC were statistically significant $(P<0.05)$, indicating that dendritic cells significantly affected prognosis and warrant further study and exploration (Fig. 5B). 
A
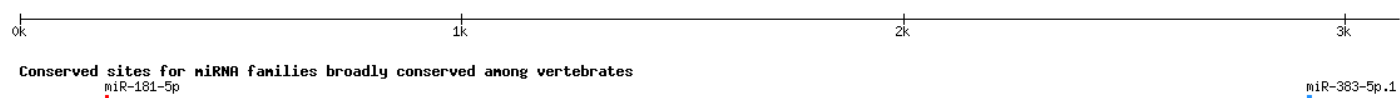
miR-181-5p

miR-383-5p.1

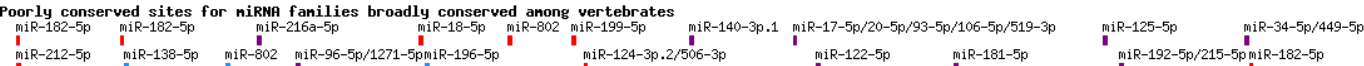

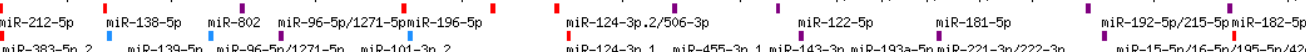

miR-383-5p.2 miR-139-5p miR-96-5p/1271-5p miR-101-3p.2 miiR-124-3p.1 miR-455-3p.1 miR-143-3p miR-193a-5p miR-221-3p/222-3p miR-15-5p/16-5p/195-5p/424-5p/497-5p

miR-208-3p miR-338-3p miR-142-5p miR-802

miR-26-5p miR-190-5p

miR-499a-5p miR-216b-5p miR-181-5p

miR-183-5p.2 miR-221-3p/222-3p

min-200bc-3p/429

miR-183-5p.2

miR-182-5p

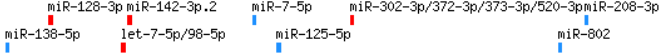

miR-23-3p "miR-142-5p | miR-499a-5p

$i^{m i R-135-5 p}$

miR-31-5p

B
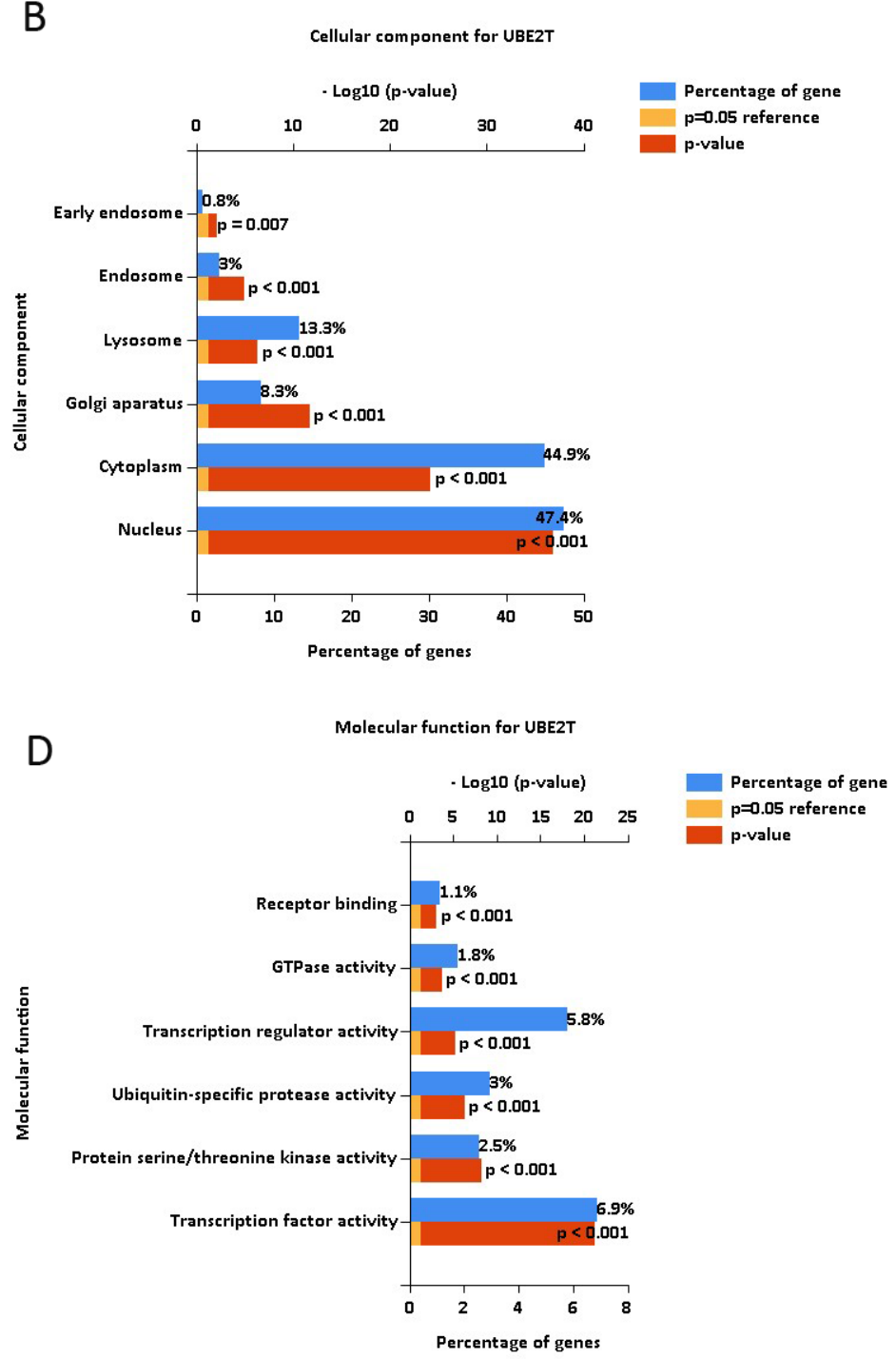

C

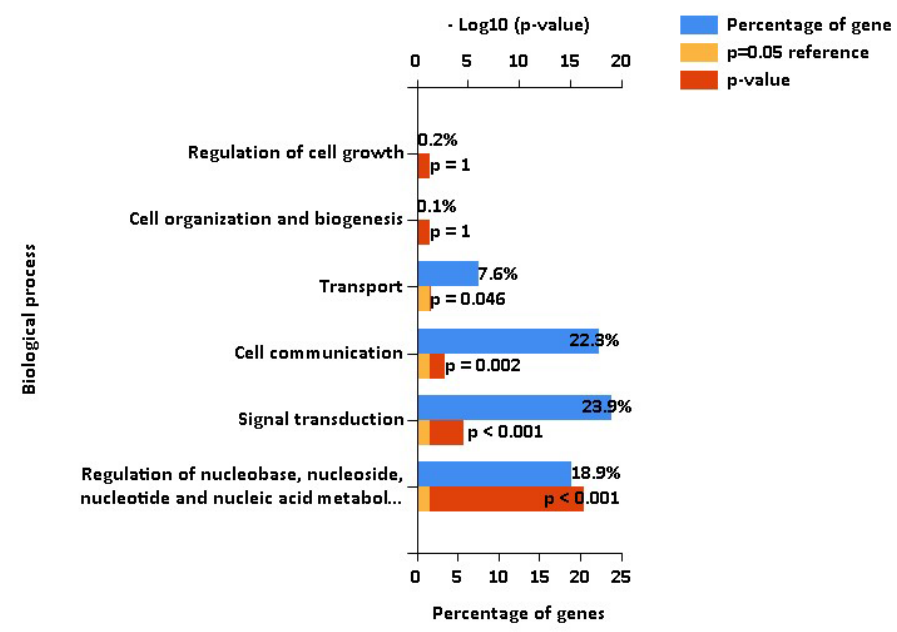

$E$

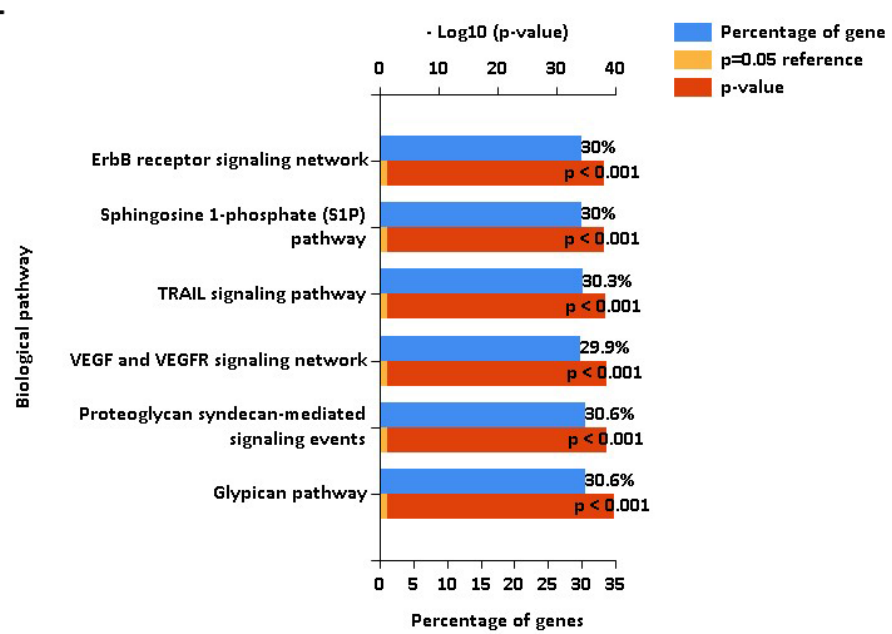

Figure 6

A box plot was introduced to demonstrate the distribution of each immunization subgroup in each copy number state with UBE2T in OC. According to the cumulative weighted context ++ score, the top three of the 1169 miRNA families associated with the gene UBE2T in OC are hsa-miR-5580-3p, hsa-miR-3652 and hsa-miR-4430. Fig. 6A shows conserved sites of the widely conserved miRNA family in vertebrates. To examine the function of the identified 1169 miRNAs, bioconcentration was performed by the Funrich 
database. Biological processes are significantly rich in nucleobase regulation, signal transduction, cellular communication, transport, cell growth, and regulation of cellular tissue and biogenesis. Cellular components are mainly enriched in the nucleus, cytoplasm, Golgi apparatus, endosomes, lysosomes and early endosomes. Molecular functions are mainly enhanced by transcription factor activity, transcriptional regulatory activity, protein serine, GTPase activity and ubiquitin-specific protease activity; biological pathways are abundant, including Glypican pathway, proteoglycan syndecan-mediated signaling events, VEGF and VEGFR signals Network, TRAIL signaling pathway, sphingosine 1-phosphate (S1P) pathway and ErbB signaling pathway Fig. 6B-6E. 